\title{
How do genetic relatedness and spatial proximity shape African swine fever infections in wild boar?
}

\author{
Tomasz Podgórski ${ }^{1}$, Kim Pepin $^{2}$, Anna Radko $^{3}$, Angelika Podbielska $^{3}$, Magdalena Eyjak ${ }^{4}$, \\ and Grzegorz Wozniakowski ${ }^{5}$ \\ ${ }^{1}$ Instytut Biologii Ssakow Polskiej Akademii Nauk \\ ${ }^{2}$ USDA-APHIS National Wildlife Research Center \\ ${ }^{3}$ Instytut Zootechniki Panstwowy Instytut Badawczy \\ ${ }^{4}$ Panstwowy Instytut Weterynaryjny - Panstwowy Instytut Badawczy w Pulawach \\ ${ }^{5}$ Uniwersytet Mikolaja Kopernika w Toruniu Wydzial Filozofii i Nauk Spolecznych
}

July 10, 2021

\begin{abstract}
The importance of social and spatial structuring of wildlife populations for disease spread, though widely recognized, is still poorly understood in many host-pathogen systems. In particular, system specific kin relationships among hosts can create contact heterogeneities and differential disease transmission rates. Here, we investigate how distance-dependent infection risk is influenced by genetic relatedness in a novel wild boar ( Sus scrofa) - African swine fever (ASF) system. We hypothesized that the infection risk would correlate positively with proximity and relatedness to ASF-infected individuals but expected those relationships to weaken with distance between individuals due to decay in contact rates and genetic similarity. ASF infection risk was shaped by the number of infected animals throughout the zone of potential contact $(0-10 \mathrm{~km})$ but not beyond it. This effect was the strongest at close distances $(0-2 \mathrm{~km})$ and weakened further on $(2-10 \mathrm{~km})$, consistent with decreasing probability of contact. Overall, there was a positive association between genetic relatedness to infectees and infection risk within the contact zone but this effect varied in space. In the high-contact zone $(0-2 \mathrm{~km})$, infection risk was not influenced by relatedness when controlled for the number of ASF-positive animals. However, infections were more frequent among close relatives indicating that familial relationships could have played a role in ASF transmission. In the medium-contact zone $(2-5 \mathrm{~km})$, infection risk and frequency of paired infections were associated with relatedness. Relatedness did not predict infection risk in low- and no-contact zones (5-10 and $>10 \mathrm{~km}$, respectively). Together, our results indicate that the number of nearby infected individuals overrides the effect of relatedness in shaping ASF transmission rates which nevertheless can be higher among close relatives. Highly localized transmission highlights the possibility to control the disease if containment measures are employed quickly and efficiently.
\end{abstract}

How do genetic relatedness and spatial proximity shape African swine fever infections in wild boar?

Tomasz Podgórski ${ }^{1,2,}$ *, Kim M. Pepin ${ }^{3}$, Anna Radko $^{4}$, Angelika Podbielska ${ }^{4}$, Magdalena Lyjak ${ }^{5}$, Grzegorz Woźniakowski ${ }^{6}$

${ }^{1}$ Mammal Research Institute, Polish Academy of Sciences, Stoczek 1, 17-230 Białowieża, Poland

${ }^{2}$ Department of Game Management and Wildlife Biology, Faculty of Forestry and Wood Sciences, Czech University of Life Sciences, Kamýcká 129, 16500 Praha 6, Czech Republic

${ }^{3}$ National Wildlife Research Center, USDA, APHIS, Wildlife Services, 4101 Laporte Ave., Fort Collins, CO, 80526 
${ }^{4}$ Department of Animal Molecular Biology, National Research Institute of Animal Production, Krakowska 1, 32-083 Balice, Poland

${ }^{5}$ Department of Swine Diseases, National Veterinary Research Institute, Partyzantów 57, 24-100 Pulawy, Poland

${ }^{6}$ Deparment of Diagnosis and Clinical Sciences, Faculty of Biological and Veterinary Sciences, Nicolaus Copernicus University, Lwowska 1,87-100 Torun, Poland

*Corresponding author: Tomasz Podgórski, Department of Game Management and Wildlife Biology, Faculty of Forestry and Wood Sciences, Czech University of Life Sciences, Kamýcká 129, 16500 Praha 6, Czech Republic;

podgorski@fld.czu.cz

\section{Summary}

The importance of social and spatial structuring of wildlife populations for disease spread, though widely recognized, is still poorly understood in many host-pathogen systems. In particular, system specific kin relationships among hosts can create contact heterogeneities and differential disease transmission rates. Here, we investigate how distance-dependent infection risk is influenced by genetic relatedness in a novel wild boar (Sus scrofa) - African swine fever (ASF) system. We hypothesized that the infection risk would correlate positively with proximity and relatedness to ASF-infected individuals but expected those relationships to weaken with distance between individuals due to decay in contact rates and genetic similarity.

ASF infection risk was shaped by the number of infected animals throughout the zone of potential contact $(0-10 \mathrm{~km})$ but not beyond it. This effect was the strongest at close distances $(0-2 \mathrm{~km})$ and weakened further on $(2-10 \mathrm{~km})$, consistent with decreasing probability of contact. Overall, there was a positive association between genetic relatedness to infectees and infection risk within the contact zone but this effect varied in space. In the high-contact zone $(0-2 \mathrm{~km})$, infection risk was not influenced by relatedness when controlled for the number of ASF-positive animals. However, infections were more frequent among close relatives indicating that familial relationships could have played a role in ASF transmission. In the medium-contact zone (2-5 $\mathrm{km}$ ), infection risk and frequency of paired infections were associated with relatedness. Relatedness did not predict infection risk in low- and no-contact zones (5-10 and $>10 \mathrm{~km}$, respectively). Together, our results indicate that the number of nearby infected individuals overrides the effect of relatedness in shaping ASF transmission rates which nevertheless can be higher among close relatives. Highly localized transmission highlights the possibility to control the disease if containment measures are employed quickly and efficiently.

Key words: ASF, disease ecology, kinship, social behavior, transmission, wild boar

\section{Introduction}

Spatial and social behavior of the hosts play major role in shaping patterns of pathogen spread in animal populations (Albery et al., 2021; Altizer et al., 2003; Dougherty et al., 2018; Sah et al., 2018). Host movements define the spatial dimension of pathogen transmission while social structure determines the encounter rate between infected and susceptible individuals. In social systems with stable group membership, individual contacts and pathogen transmission occur mainly within social groups, potentially limiting the speed of disease spread (Pepin et al., 2020). This type of social structure is typically based on familial (e.g. matrilineal) groups where social interactions and disease transmission rates are correlated with genetic relatedness (Carter et al., 2013; Grear et al., 2010). In contrast, in fission-fusion societies with dynamic group membership, contact rates and disease transmission tend to be independent of relatedness (Hirsch et al., 2013; MejíaSalazar et al., 2017; Vander Wal et al., 2012). Social system can thus affect the rate and mechanisms of pathogen transmission (Altizer et al., 2003; Sah et al., 2018). Contact heterogeneity due to social structure may be particularly relevant for disease transmission at a local scale, where groups are already in spatial proximity allowing for contact. Understanding relative contribution of spatial and social process to disease transmission at such scales is important for modeling and managing wildlife diseases (Dougherty et al., 
2018; Pepin et al., 2021). However, investigating the role of host social and spatial behavior in disease transmission is challenging, ideally requiring simultaneous host contact, movement and infection data and studies addressing this issue with relevant data are limited. Additionally, host social system and pathogen characteristics (e.g. infectiousness, transmission mode, lethality) interact to produce varying spatial infection patterns and epidemiological outcomes (Pepin and VerCauteren, 2016; VanderWaal and Ezenwa, 2016).

African swine fever (ASF) is a contagious viral disease with both direct and environmental transmission in Eurasian wild boar Sus scrofawhich is the sole reservoir of the disease in Eastern Europe (Chenais et al. 2018). The ASF virus strain currently circulating in Eastern Europe (genotype II) is highly virulent, causing lethality approaching 100\% within 1-3 weeks post-infection (Blome et al., 2013) and the disease causes high mortality in the susceptible host populations which can reduce wild boar numbers by as much as $90 \%$ during the initial phase of an outbreak (Morelle et al., 2020). ASF virus is resistant to environmental factors and can remain active in contaminated tissues from several weeks to months (Fischer et al., 2020). Transmission through infected carcasses has been estimated to account for about a half of all infections and contribute to long-term persistence of the disease particularly at low host densities (Pepin et al., 2020). Because diseased animals tend to die locally (i.e. within their home range), they will be a source of infections mainly for the most proximate individuals from their own or neighboring social groups. However, transmission rates via indirect and direct (i.e. through social interactions) routes will probably differ due to varying contact dynamics and long availability of infectious carcasses (Cukor et al., 2020; Probst et al., 2017; Probst et al., 2020). Thus, carcass-based transmission interacts with direct transmission to shape local infection patterns (Lange and Thulke, 2016). The spread of infectious diseases occurs over multiple spatial scales (Riley, 2007). On the landscape level, ASF prevalence and spread correlate positively with wild boar density (Nurmoja et al., 2017; Podgórski et al., 2020), proportion of forest cover (Dellicour et al., 2020; Podgórski et al., 2020) and negatively with distance to previous cases (Podgórski et al., 2020) and physical barriers to wild boar movement (Dellicour et al., 2020). At fine scales, ASF transmission is likely influenced by a combination of social interactions, movements, and spatial distribution of individuals (Pepin et al., 2021).

Wild boar social structure is based on cohesive, matrilineal social units (Gabor et al., 1999; Kaminski et al., 2005; Podgórski et al., 2014b). Contact rates are strongly structured socially and spatially (Pepin et al., 2016; Podgórski et al., 2018; Yang et al., 2020). The rate of inter-group interactions is relatively low and declines sharply with distance between the groups. The highest contact rates are between immediately adjacent groups $(0-2 \mathrm{~km})$ and drop to very low levels at a distance as close as $4 \mathrm{~km}$. Such type of social structure is not conducive to rapid spread of infectious diseases (Pepin and VerCauteren, 2016). Social behavior of wild boar, next to its sedentary lifestyle, is probably one of the factors responsible for slow natural spread of ASF in wild boar populations. While wild boar movements were shown to be poor predictors of ASF spread (Podgórski and Smietanka, 2018), the role of genetic relatedness as a predictor of social interaction rates has received little attention as a potential driver of ASF transmission. A recent model of ASF transmission in wild boar highlighted a significant role of social structure in shaping spatial and temporal dynamics of ASF spread and showed that most transmission events occurred within family groups and within close distance $<1.5 \mathrm{~km}$ (Pepin et al., 2021). However, real time infection and contact tracing data that could validate predictions from models of surveillance data are notoriously difficult to obtain from field studies and no such data exist for the wild boar - ASF system. Here we use genetic relatedness as a proxy of social interactions as those two have been shown to correlate in the kin-based wild boar society (Podgórski et al., 2014b). Kinship has been shown to predict infection risk in other wildlife disease systems, e.g. chronic wasting disease in white-tailed deer (Grear et al., 2010) or bovine tuberculosis in badgers (Benton et al., 2016).

Previous studies have shown that probability of ASF occurrence in wild boar populations increases with proximity to previous cases at a coarse spatial scale ( $>10$ kilometers) (Podgórski et al., 2020), while transmission rates appear to be the highest at fine scales ( $<2$ kilometers (Pepin et al., 2021). Here, we investigate whether distance-dependent infection risk is influenced by genetic relatedness at a local spatial scale where relatedness might influence contact structure and thus impact disease transmission. Because genetic relatedness and distance between hosts are correlated, we designed the analysis to examine the role of genetic relatedness among hosts within fixed spatial distances among hosts ('proximity'), with the proximity ca- 
tegories based on previous work of wild boar spatial contact ecology (see below). We hypothesized that the infection risk would correlate positively with proximity (H1, Table 1) and relatedness (H2, Table 1) to ASF-positive individuals. We expected relationships of infection risk and proximity or genetic relatedness to become weaker with increasing distance between individuals due to decay in contact rates (Pepin et al., 2016; Podgórski et al., 2018) and genetic similarity (Podgórski et al., 2014a; Poteaux et al., 2009) (H3, Table 1). Based on current knowledge of wild boar socio-spatial ecology, we analysed infection risk in four distance classes: 1) 'high-contact' zone $(0-2 \mathrm{~km})$ : social contacts among individuals are most frequent, both within and between groups (Podgórski et al., 2018; Yang et al., 2020) , 2) 'medium-contact' zone (2-5 km): interactions among neighbouring social groups (Pepin et al., 2016; Podgórski et al., 2018) , 3) 'low-contact' zone (5-10 km): sporadic contacts between distant groups with non-overlapping home ranges, distance of most natal dispersal (Keuling et al., 2010; Podgórski et al., 2014a; Prévot and Licoppe, 2013), 4) 'no-contact' zone $(>10 \mathrm{~km}$ ): groups do not interact, occasional long-distance movements (Andrzejewski and Jezierski, 1978; Podgórski et al., 2014a). We predicted that variation in infection risk would be explained by relatedness and proximity to infected individuals in high-contact and medium-contact zones (P3.1). In the low-contact zone infection risk would be predicted by the distance to infectees but not by relatedness to them (P3.2.). In the no-contact zone, neither relatedness nor proximity would shape infection risk (P3.3).

Table 1. Hypotheses and corresponding predictions on relationships between proximity and relatedness to ASF-positive individuals and infection risk.

\begin{tabular}{ll}
\hline Hypotheses & Predictions \\
\hline $\begin{array}{l}\text { H1. Infection risk increases with proximity to } \\
\text { infected individuals due to higher chances of } \\
\text { contact }\end{array}$ & $\begin{array}{l}\text { P1.1: positive and strongest effect of the proportion } \\
\text { of infected individuals in the 'high-contact' (0-2km) } \\
\text { P1.2: positive but weaker effects of the proportion } \\
\text { of infected individuals in 'medium-contact' (2-5km) } \\
\text { and 'low-contact' (5-10km) zones P1.3: no effect of } \\
\text { the proportion of infected individuals in the } \\
\text { 'no-contact' ( }>10 \mathrm{~km}) \text { zone }\end{array}$ \\
$\begin{array}{l}\text { P2.1: positive effect of relatedness to infected } \\
\text { individuals across contact zones (0-10km) P2.2: no } \\
\text { effect of relatedness to infected individuals in the }\end{array}$ \\
$\begin{array}{l}\text { H2. Infection risk increases with relatedness to } \\
\text { infected individuals due to kin-based social } \\
\text { structure }\end{array}$ & $\begin{array}{l}\text { P3.1: positive effect of relatedness and proportion } \\
\text { of infected individuals in the 'high-contact' and } \\
\text { potentially 'medium-contact' zones P3.2: positive } \\
\text { effect of the proportion of infected individuals and } \\
\text { decline with distance to infected individuals due the } \\
\text { nocial structure and spatial clustering }\end{array}$ \\
$\begin{array}{l}\text { no effect of relatedness in the 'low-contact' zone } \\
\text { infected individuals in the 'no-contact' zone }\end{array}$ \\
\hline
\end{tabular}

\section{Materials and methods}

\section{Study area and sample collection}

The study was conducted in north-eastern Poland where ASF was introduced in February 2014, $<1 \mathrm{~km}$ from the border with Belarus, and has subsequently spread through the region in wild boar (Podgórski et al., 2020) and domestic pigs (Taylor et al., 2021). By mid 2016, ASF cases in wild boar were distributed continuously in the infected region covering about $4500 \mathrm{~km}^{2}$ and this is where samples used in this study originate (Fig. 1). The area is mostly field-woodland mosaic characterized by extensive agriculture and low human population density (60 people $/ \mathrm{km}^{2}$ ) and relatively high forest cover (31\%). The landscape is flat (highest elevation $298 \mathrm{~ms}$ a.s.l.) with no significant natural or anthropogenic barriers to wild boar movement. Wild boar are 
distributed continually throughout the area with densities at the start of the ASF epidemic ranging between 0.5 and 5 ind. $/ \mathrm{km}^{2}$ at the forest district level (Regional Directorate of State Forests, Białystok, Poland).

Following the introduction of ASF, an intensive surveillance program was implemented in the affected area. The program conducted laboratory tests of all hunted wild boar (active surveillance) and all wild boar found dead (passive surveillance). We used surveillance data routinely collected by the National Reference Laboratory for ASF at the National Veterinary Research Institute in Puławy, Poland. Wild boar samples were classified as ASF-positive (herefater "case") if presence of viral DNA was confirmed in real-time PCR or antibodies were detected using ELISA test and confirmed with a immunoperoxidase test (IPT). Detailed description of the surveillance design and laboratory procedures can be found in (Woźniakowski et al., 2016). A total of 5487 wild boars were sampled in the study area from February 2014 to July 2016 and 168 of them tested positive for ASF. It was not technically possible to genotype all of the sampled individuals. Instead, we selected a subsample of 1078 individuals (including all ASF-positive) evenly distributed across the study area for further analyses. However, many samples, particularly originating from the carcasses, were of poor quality and yielded too few microsatellite loci (those yielding less than 13 out of 16 loci were excluded). Finally, we were able to satisfactorily genotype 323 samples (including 80 ASF cases) which comprised a final dataset.

\section{Microsatellite genotyping}

DNA was extracted from tissue and blood using the Sherlock AX Kit (A\&A Biotechnology, Gdynia, Poland), following the manufacturer's protocol. The extracts were quantified with a NanoDrop 2000 spectrophotometer (Thermo Scientific, Wilmington, DE, USA). All individuals were genotyped with a panel of 16 polymorphic microsatellite loci (S090, SW72, S155, S026, S355, S215, SW951, SW857, SW24, SW122, IGF1, SW461, SW1492, SW2021, SW2496, SW2532), which had been successfully used to study relatedness and genetic variation in wild boar populations from the study area (Podgórski et al., 2014a; Podgórski et al., 2014b). The 16 autosomal STR loci were amplified in two independent multiplexed mixes using Qiagen Master Mix (Qiagen Inc, Hilden, Germany) reagents and fluorescently label primers. For the first panel, the fluorochromes were used: 6-FAM for the loci SW72, SW857; VIC for SW1492, IGF1; NED for S026, S215; PET for SW2021, SW2532 and for second panel 6-FAM for SW122, S0355; VIC for SW461, SW2496; NED for W24, S0155 and PET for SW951 and S0090. PCR was performed on Veriti@) Thermal Cycler amplifier (Applied Biosystems, Foster City, CA, USA) using the following thermal profile: $5 \mathrm{~min}$. of initial DNA denaturation at $95^{\circ} \mathrm{C}$, followed by 35 cycles of denaturation at $95^{\circ} \mathrm{C}$ for $30 \mathrm{~s}$, annealing at $56^{\circ} \mathrm{C}$ for $90 \mathrm{~s}$, elongation of starters at $72^{\circ} \mathrm{C}$ for $30 \mathrm{~s}$ and final elongation of starters at $60^{\circ} \mathrm{C}$ for $30 \mathrm{~min}$. Analysis of the obtained PCR products was performed using an ABI 3130xl capillary sequencer ( Applied Biosystems, Foster City, CA, USA) at the Institute of National Research Institute of Animal Production (Cracow, Poland). The amplified DNA fragments were subjected to electrophoresis in 7\% denaturing POP-7 polyacrylamide gel in the presence of a standard length of $500 \mathrm{Liz}$ and a reference sample. The results of the electrophoretic separation were analysed automatically using the GeneMapper $($ S Software 4.0 ( Applied Biosystems, Foster City, CA, USA).

\section{Analysis of relatedness and spatial-genetic structure}

Basic parameters of microsatellite polymorphism and genetic diversity were calculated using GENALEX 6.5 (Peakall \& Smouse, 2006) and FSTAT (Goudet, 1995). GENEPOP 4.7 (Raymond \& Rousset, 1995) was used to test loci for departures from linkage equilibrium and Hardy-Weinberg equilibrium (HWE) using the

Markov chain method (parameters: 1000 dememorization steps, 100 batches, 1000 iterations per batch). The significance level was adjusted for multiple testing across loci using the sequential Bonferroni's correction (Rice, 1989).

Pairwise genetic relatedness (Queller and Goodnight, 1989) was calculated with GENALEX 6.5 among all individuals $(\mathrm{n}=323)$ and subsequently used in all analyses. The Queller and Goodnight estimator is an unbiased measure of relatedness based on population allele frequencies. It ranges from -1 to 1 , where positive values indicate pairs more related than average and negative less related than average. We used the 
Queller and Goodnight estimator to allow comparison with previous studies of wild boar social structure which we build upon (Podgórski et al., 2014a) . We used a dyadic relatedness as continuous predictor in models explaining variation in infection probability. We also dychotomized highly related (hereafter "kin") and unrelated (herefater "non-kin") pairs of individuals using previously published mean relatedness between social group members of 0.247 (Podgórski et al., 2014a). Analysis of spatial-genetic structure was performed with GENALEX 6.5. Autocorrelation coefficients $(r)$ between pairwise genetic and geographic distance matrices were calculated for pre-defined Euclidean distance classes (Supplementary Information: Fig. S1) which correspond to spatial genetic structure previously observed in wild boar populations (Pepin et al., 2016; Podgórski et al., 2014a; Podgórski et al., 2018; Poteaux et al., 2009). Spatial-genetic structure was examined among all individuals as well as among ASF-positive and ASF-negative individuals separately. Spatial autocorrelation coefficients were compared across distance classes using randomization tests (10 000 permutations) (Manly 1997). When estimating probability of infection and comparing relatedness within distance classes, we merged the $10+\mathrm{km}$ classes into one due to weak genetic structure between pairs and negligible contact rates beyond $10 \mathrm{~km}$. We compared relatedness of individuals with paired infections (pairs where both individuals were infected) and with no paired infection (none or one individual of the pair were infected) within the same distance classes as in models estimating probability of infection $(0-2 \mathrm{~km}$, $2-5 \mathrm{~km}, 5-10 \mathrm{~km},>10 \mathrm{~km}$ ) and using the same temporal windows of potential transmission to select pairs for comparison. Relatedness levels were compared using randomization tests (10 000 permutations) (Manly 1997).

\section{Estimating probability of infection}

For every sampled individual $(\mathrm{n}=323)$, we selected ASF-positive individuals that could have been the source of infection using a temporal window (30 days) that reflects the upper limit of lifespan after ASF infection, i.e. infection-to-death time (Gallardo et al., 2017; Pietschmann et al., 2015). For samples originating from carcasses, the window was set to 60 days prior to sampling and included infection-to-death time and carcass decomposition time during which the sample was taken. A decomposition time of 30 days was chosen arbitrarily because the decomposition status of most carcasses was unknown and the exact date of death could not be determined. This time frame was conservative given usually longer decomposition times of wild boar tissues (Probst et al., 2020) and ASF virus persistence in them (Fischer et al., 2020). Additionally, the temporal window was extended by 7 days post-sampling of the focal individual to account for the retrieval and reporting lag of the ASF-positives. ASF-positive individuals selected within those time frames were then categorized into four distance classes $(0-2 \mathrm{~km}, 2-5 \mathrm{~km}, 5-10 \mathrm{~km},>10 \mathrm{~km})$. The proportion of ASF cases in each distance class were used as covariates explaining the binary response of ASF infection status of the focal individual (0 - negative, 1- positive).

We analysed the effects of explanatory variables on the probability of ASF infection using a generalized linear model with a binomial response and "logit" link function (Zuur, 2009). The probability of ASF infection reflects the likelihood of any sampled individual from the study area testing positive for ASF within the 2.5 years of the study period based on the covariates. Proportion of ASF-positive individuals within the four aforementioned distance classes and relatedness to ASF-positive individuals within 0-10 km was used as covariates to verify the predictions (Table 1). Sample sizes across covariate categories were unbalanced and precluded formulation of a global model including all explanatory variables. Therefore, the structure of the models was developed progressively, starting with simple formulations where each model included just one covariate explaining infection risk (Supplementary Information: Table S1). We then contrasted, in four separate models (Table 2), information on relatedness to ASF-positive individuals within 0-10 km with the proportion of ASF-positive individuals in each of the four distance classes. This approach allowed us to test the relative effect of relatedness and spatial proximity to infectees on infection risk while accounting for the greater number of highly related individuals at close distances resulting from wild boar social structure. Explanatory variables did not show significant collinearity (variance inflation factors - VIFs $<1.3$ for all models). We did not perform model selection for reduced models within each hypothesis as we were interested in testing specific predictions represented by each of the candidate explanatory variables in our full models and we could not compare across models because the response variable was different for each model. We 
quantified variation explained by the fixed effects of the model by calculating marginal $R^{2}$ GLMM (Nakagawa et al., 2017) using the MuMIn R-package (Barton 2020). All statistical analyses were performed in R 4.0.2 (R Core Team 2020). The models were computed using the "lme4" package (Bates et al. 2015).

\section{Results}

\section{Spatial-genetic structure and relatedness}

In total, 135 alleles were detected across 16 analysed loci. All loci were polymorphic with the number of alleles per locus ranging from 3 to 18 (mean \pm SE: $8.4 \pm 0.94$ ). Missing data (i.e. $\%$ of missing alleles) amounted to $2.4 \%$ of the dataset and no individual was typed at less than 13 loci. Expected and observed heterozygosity averaged $0.61 \pm 0.06$ and $0.56 \pm 0.06$, respectively. Following sequential Bonferroni's corrections, the overall population showed deviation from HWE at eight loci and from linkage equilibrium in 2 out of 120 pairs of loci. Such deviation from equilibrium was most likely attributed to the inherent substructure of the population (i.e. presence of kin groups) and all loci were retained for statistical analyses.

Spatial autocorrelation analysis revealed the presence of genetic structure, as indicated by an autocorrelation coefficient $(r)$ greater than at random, at a distance of up to $20 \mathrm{~km}$ at the population level and up to 10 $\mathrm{km}$ among ASF-positive individuals, with genetic similarity between individuals declining from the smallest distance (Fig. S1). Genetic structuring was stronger among ASF-positive individuals compared to ASFnegative ones within each distance class up to $10 \mathrm{~km}\left(0-2.5 \mathrm{~km}\right.$ distance class: $r_{\text {pos }}=0.078 \pm 0.003$ and $r_{\text {neg }}$ $=0.033 \pm 0.001, \mathrm{p}<0.001 ; 2.5-5 \mathrm{~km}$ distance class: $r_{\text {pos }}=0.028 \pm 0.002$ and $r_{\text {neg }}=0.015 \pm 0.001, \mathrm{p}<$ $0.001 ; 5-10 \mathrm{~km}$ distance class: $r_{\text {pos }}=0.019 \pm 0.001$ and $r_{\text {neg }}=0.011 \pm 0.0004, \mathrm{p}<0.001 ;$ Fig. S1).

Considering the entire study area, the coefficient of relatedness in the population averaged $0.0003 \pm 0.002$ and relatedness among individuals with paired infections was much higher than among those without paired infections $(0.049 \pm 0.009$ and $-0.003 \pm 0.002$, respectively, $\mathrm{p}<0.001)$. The differences were influenced by the distance between pairs (Fig. 2). Relatedness among animals with paired infections was highest in the 0-2 $\mathrm{km}$ and $2-5 \mathrm{~km}$ distance classes $(0.129 \pm 0.054$ and $0.129 \pm 0.055$, respectively) and lower in the subsequent classes (5-10 km: $0.018 \pm 0.017 ;>10 \mathrm{~km}: 0.042 \pm 0.009)$. Relatedness between individuals with paired infections was consistently higher than among those without paired infections, except within the $5-10 \mathrm{~km}$ distance class (Fig. 2).

\section{Probability of infection}

We found a positive relationship between the probability of ASF infection and the proportion of infected individuals in high-, medium-, and low-contact zones $(0-2 \mathrm{~km}, 2-5 \mathrm{~km}$, and $5-10 \mathrm{~km}$, respectively) (Supplementary Information, Table S1, Fig. S2). The strongest effect of the proportion of infectees was observed in the high-contact zone, where infection risk increased from $2.5 \%$ if no infected individuals were present to $96 \%$ if all other individuals were ASF-positive. Proportion of infected individuals at distances over 10 $\mathrm{km}$ did not influence the probability of infection (Table S1, Fig. S2). Relatedness was a positive predictor of infection risk within $10 \mathrm{~km}$ but had no effect at larger distances (Table S1, Fig. S2). Probability of infection among kin (i.e. presumably group members) across the study area was on average $77 \pm 1.5 \%$ (min-max: 69-87) within $10 \mathrm{~km}$, while infection risk among non-kin averaged $52 \pm 0.9 \%$ (min-max: 28-68) within $10 \mathrm{~km}$ (Fig. S2a). Models combining relatedness and proximity to infected individuals (Table 2) showed that the relative contribution of relatedness and proportion of infected individuals to explaining variation in infection probability varied with distance. In the high-contact zone, variation in infection risk was shaped overwhelmingly by the proportion of infected individuals and not by relatedness (Table 2, Fig 3). However, infection risk among kin within $2 \mathrm{~km}$ distance was high. All of the animals which were surrounded by ASF-positive kin $(\mathrm{n}=17)$ tested positive, while none of the animals surrounded by ASF-negative kin $(\mathrm{n}=7)$ tested positive. Among animals surrounded only by infected non-kin between 0 and $2 \mathrm{~km}(\mathrm{n}=18)$, the proportion of ASF-positive individuals was $83 \%$, indicating they were $17 \%$ less likely to become infected by non-kin relative to kin on average. At further distances of $2-5 \mathrm{~km}$ (medium-contact zone) infection risk increased with both relatedness and proportion of ASF-positive individuals at this distance (Table 2, Fig.3), indicating that ASF transmission was significantly more likely between related individuals. In the medium-contact zo- 
ne, infection risk averaged $84 \pm 3.5 \%$ (min-max: 58-93) among kin and $47 \pm 2.5 \%$ (min-max: 16-81) among non-kin. At even further distances of 5-10 km (low-contact zone), probability of infection increased with the number of ASF-positive individuals while relatedness to infectees did not shape infection risk. Here, infection risk increased from $38 \%$ if at least one infected individual was present to $75 \%$ if all other individuals were ASF-positive (Fig. 3). At distances $>10 \mathrm{~km}$ (no-contact zone), neither relatedness to infected individuals nor their number influenced infection risk (Table 2, Fig.3).

\section{Discussion}

Our results showed that the risk of ASF infection was shaped by interacting effects of spatial proximity and genetic relatedness to infected individuals. Proximity and number of infected animals had consistently positive effect on infection risk throughout the zone of potential contact $(0-10 \mathrm{~km})$ but not beyond it, thus fully supporting H1. The number of ASF-positive individuals had the strongest effect on infection risk in the high-contact zone $(0-2 \mathrm{~km}$; P1.1), weaker but similar within medium- $(2-5 \mathrm{~km})$ and low-contact $(5-10 \mathrm{~km})$ zones (P1.2), and no effect in the no-contact zone (>10 km; P1.3). There was a positive association between genetic relatedness to infectees and infection risk within the contact zone $(0-10 \mathrm{~km})$, supporting $\mathrm{H} 2$, but this effect varied in space (H3). In the high-contact zone, infection risk was not influenced by relatedness when controlled for the number of ASF-positive animals. However, infections were more frequent among close relatives, i.e. kin or group members, indicating that familial relationships could have played a significant role in ASF transmission. In the medium-contact zone, infection risk was associated with relatedness and paired infections were more frequent among relatives. For the most part, those results provide support of P3.1. Relatedness did not predict infection risk in low- and no-contact zones (P3.2 and P3.3, respectively).

Social contacts directed towards relatives can lead to increased pathogen transmission within familial groups and local clustering of disease prevalence (Blanchong et al., 2007; Delahay et al., 2000). Social relationships in wild boar tend to be the strongest among closely related group members (Gabor et al., 1999; Podgórski et al., 2018; Podgórski et al., 2014b). Therefore, we predicted a strong effect of relatedness on disease transmission at close distances $(0-2-\mathrm{km})$ due to socially-driven contact heterogeneity. This prediction was not supported in our regression model possibly because at this distance most individuals are highly related and there are not enough unrelated individuals to find statistical significance. However, our descriptive analysis found that individuals which were infected simultaneously (i.e. paired infections) tended to be more related than those uninfected. This trend was particularly noticeable at the upper range of relatedness distribution, i.e. among close kin or group members. Such a pattern is consistent with kin-biased associations in wild boar, particularly among females and young animals, manifested in more regular and longer lasting contacts (Podgórski et al., 2014b; Poteaux et al., 2009) which facilitate disease transmission. Similarly, kinship was shown to drive bovine tuberculosis infections in badger cubs exposed to infectious females in a natal sett (Benton et al., 2016). Inter-group contacts in wild boar occur most frequently at a spatial scale similar to interactions within groups (Podgórski et al., 2018; Yang et al., 2020). This spatial overlap in social connectivity may confound the effects of inter- vs. intra-group transmission. Using relatedness as an indicator of group membership could potentially help untangle those effects. However, in our study the number of ASF-positive individuals in close vicinity was a much stronger predictor of infection risk compared to relatedness. It thus appears that at close distances kin relationships do not have a strong impact on structuring contacts and shaping ASF transmission beyond the closest relatives. Our analysis did not discriminate between infections originating from the contact with infectious carcass and infected live animals. Therefore, it is possible that indirect transmission through infected carcasses and contaminated environment (Chenais et al. 2018) contributed significantly to recorded infections. In fact, indirect transmission accounts for more than half of ASF infections in wild boar population (Pepin et al., 2020). Animals could have come into contact with nearby infected carcass of the individual which was a member of a different but spatially overlapping social group, i.e. was not closely related. Abundance of infectious carcasses in the surrounding environment could thus lead to infection regardless of relatedness and produce confounding effects that masked actual transmission contacts among live animals.

Interestingly, infection risk correlated with relatedness in the medium-contact zone $(2-5 \mathrm{~km})$ suggesting that 
social connectivity with relatives extended beyond the closest group members. This pattern is unlikely to have resulted from the dispersing individuals infected in the natal groups because it typically takes longer to disperse (Podgórski et al., 2014a) than it takes the disease to hamper movements (Blome et al., 2013). To alleviate symptoms of the disease, infected wild boar seek specific habitats which differ from those regularly used (Morelle et al., 2019). Those preferences and restricted mobility of sick animals can separate them from the group and result in dispersion of diseased group members and wide distribution of samples from related individuals. Additionally, kin-directed interactions over larger distances could be maintained by temporal fission-fusion events of core groups, similar to observed in African elephants (Archie et al., 2006) or giraffes (Carter et al., 2013). These dynamics could provide a mechanism for disease transmission among distant relatives. However, fission-fusion dynamics in wild boar has not been systematically studied and it is difficult to tell whether temporal scales of social and disease dynamics would match and help explain patterns observed in our study. Besides relatedness to infected individuals, their number had a strong impact on infection risk within the medium-contact zone which indicates that contacts between groups and/or with infected carcasses played an important role in transmission.

Relatedness did not play a major role in shaping ASF infections beyond $5 \mathrm{~km}$ distance. In the low-contact zone $(5-10 \mathrm{~km})$, infection risk was influenced only by the number of infected individuals. It is unlikely that direct transmission played a significant role in producing this effect since inter-group contacts are very rare at those distances (Pepin et al., 2016; Podgórski et al., 2018). While a distance of 5-10 km exceeds the size of typical home range, it is within a range of daily travel (Podgorski et al., 2013) and could be covered during dispersal, foraging or mating forays leading to distant transmission events. Those behaviors, however, are not typically seeking contact with kin and particularly in case of mating or dispersal are often seeking non-kin to avoid inbreeding (Archie et al., 2007; Biosa et al., 2015; Hoffman et al., 2007). The observed effect of distant infected individuals on infection risk could be also a by-product of correlated local enzootic dynamics (i.e. spatial and temporal co-occurrence of cases) rather than direct transmission. Spatial clusters of increased ASF prevalence identified previously were measured at 20-40km (Podgórski et al., 2020; Taylor et al., 2021). However, those studies used data aggregated over time periods (months-years) exceeding temporal windows of ASF transmission (days-weeks) and thus could not capture fine-scale disease dynamics well. Our results indicate that ASF outbreaks are even more localized and with realistic transmission times do not exceed 10 $\mathrm{km}$ distance. This is supported by strong genetic structuring among infected animals (Fig. S2) and no effect of the number of infected individuals located at $>10 \mathrm{~km}$ on infection risk (Table 2, Fig.3). The spatial limits of transmission highlight the possibility to control outbreaks if containment measures, such as fencing, zoning and carcass removal, are employed immediately and early detection is ensured by effective surveillance.

Together our results show that ASF infection risk declines with distance, matching spatial changes in contact intensity. Infection-causing contacts were structured by relatedness particularly in medium- and, to lesser degree, high-contact zones. At close distances infections were more frequent only among close kin while at medium distances relatedness predicted infections risk more consistently. This indicates that physical kin relationships can extend beyond the immediate social environment and induce differential transmission rates, similarly to transmission of chronic wasting disease in white-tailed deer (Grear et al., 2010). However, infection risk was primarily influenced by the number of infected individuals throughout the high-, medium-, and low-contact zones. This effect was particularly strong at close distances conforming to the previous modeling study which found that most transmission events occur within $<1.5 \mathrm{~km}$ with some rare events at longer distances (Pepin et al., 2021). Spatially limited transmission is further supported by an insignificant effect of the number of infected individuals present at long distances $(>10 \mathrm{~km})$.

\section{Acknowledgements}

This work was supported by the National Science Centre, Poland (grant number 2014/15/B/NZ9/01933) and Ministry of Agriculture, Czech Republic (grant number

QK1910462). We thank M. Niedziałkowska from the Mammal Research Institute, Polish Academy of Sciences, for help in genetic analyses. 


\section{Conflict of interests}

Authors declare no conflict of interest.

\section{References}

Albery G.F., Kirkpatrick L., Firth J.A., Bansal S. (2021) Unifying spatial and social network analysis in disease ecology. Journal of Animal Ecology 90:45-61. DOI: https://doi.org/10.1111/1365-2656.13356.

Altizer S., Nunn C.L., Thrall P.H., Gittleman J.L., Antonovics J., Cunningham A.A., Dobson A.P., Ezenwa V., Jones K.E., Pedersen A.B. (2003) Social organization and parasite risk in mammals: integrating theory and empirical studies. Annual Review of Ecology, Evolution, and Systematics 34:517-547.

Andrzejewski R., Jezierski W. (1978). Management of a wild boar population and its effects on commercial land. Acta Theriologica 23:309-339.

Archie E.A., Moss C.J., Alberts S.C. (2006) The ties that bind: genetic relatedness predicts the fission and fusion of social groups in wild African elephants. Proceedings of the Royal Society B: Biological Sciences 273:513-522. DOI: $10.1098 / \mathrm{rspb} .2005 .3361$.

Archie E.A., Hollister-Smith J.A., Poole J.H., Lee P.C., Moss C.J., Maldonado J.E., Fleischer R.C., Alberts S.C. (2007) Behavioural inbreeding avoidance in wild African elephants. Molecular Ecology 16:4138-4148. DOI: $10.1111 / \mathrm{j} .1365-294 \mathrm{X} .2007 .03483 . x$.

Bartoń K., 2020: MuMIn: Multi-Model Inference. R package version 1.43.17. https://CRAN.Rproject.org $/$ package $=$ MuMIn

Bates, D., M. Maechler, B. Bolker, S. Walker, 2015: Fitting Linear Mixed-Effects Models Using lme4. Journal of Statistical Software, 67, 1-48. DOI: 10.18637/jss.v067.i01

Benton C.H., Delahay R.J., Robertson A., McDonald R.A., Wilson A.J., Burke T.A., Hodgson D. (2016) Blood thicker than water: kinship, disease prevalence and group size drive divergent patterns of infection risk in a social mammal. Proceedings of the Royal Society of London B: Biological Sciences 283. DOI: 10.1098/rspb.2016.0798.

Biosa D., Grignolio S., Sica N., Pagon N., Scandura M., Apollonio M. (2015) Do relatives like to stay closer? Spatial organization and genetic relatedness in a mountain roe deer population. Journal of Zoology:n/a-n/a. DOI: $10.1111 /$ jzo.12214.

Blanchong J.A., Scribner K.T., Kravchenko A.N., Winterstein S.R. (2007) TB-infected deer are more closely related than non-infected deer. Biology Letters 3:104-106. DOI: doi:10.1098/rsbl.2006.0547.

Blome S., Gabriel C., Beer M. (2013) Pathogenesis of African swine fever in domestic pigs and European wild boar. Virus Research 173:122-130. DOI: http://dx.doi.org/10.1016/j.virusres.2012.10.026.

Carter K.D., Seddon J.M., Frère C.H., Carter J.K., Goldizen A.W. (2013) Fission-fusion dynamics in wild giraffes may be driven by kinship, spatial overlap and individual social preferences. Animal Behaviour 85:385394. DOI: 10.1016/j.anbehav.2012.11.011.

Chenais E., Ståhl K., Guberti V., Depner K. (2018) Identification of Wild Boar-Habitat Epidemiologic Cycle in African Swine Fever Epizootic. Emerging Infectious Diseases 24:810-812. DOI: 10.3201/eid2404.172127.

Cukor J., Linda R., Václavek P., Mahlerová K., Šatrán P., Havránek F. (2020) Confirmed cannibalism in wild boar and its possible role in African swine fever transmission. Transboundary and Emerging Diseases 67(3): 1068-1073.

Delahay R.J., Langton S., Smith G.C., Clifton-Hadley R.S., Cheeseman C.L. (2000) The spatio-temporal distribution of Mycobacterium bovis (bovine tuberculosis) infection in a high-density badger population. Journal of Animal Ecology 69:428-441. DOI: https://doi.org/10.1046/j.1365-2656.2000.00406.x. 
Dellicour S., Desmecht D., Paternostre J., Malengreaux C., Licoppe A., Gilbert M., Linden A. (2020) Unravelling the dispersal dynamics and ecological drivers of the African swine fever outbreak in Belgium. Journal of Applied Ecology 57:1619-1629. DOI: 10.1111/1365-2664.13649.

Dougherty E.R., Seidel D.P., Carlson C.J., Spiegel O., Getz W.M. (2018) Going through the motions: incorporating movement analyses into disease research. Ecology Letters 21:588-604. DOI: https://doi.org/10.1111/ele.12917.

Fischer M., Hühr J., Blome S., Conraths F.J., Probst C. (2020) Stability of African Swine Fever Virus in Carcasses of Domestic Pigs and Wild Boar Experimentally Infected with the ASFV "Estonia 2014" Isolate. Viruses 12:1118.

Gabor T.M., Hellgren E.C., Van den Bussche R.A., Silvy N.J. (1999) Demography, sociospatial behaviour and genetics of feral pigs (Sus scrofa) in a semi-arid environment. Journal of Zoology 247:311-322. DOI: 10.1111/j.1469-7998.1999.tb00994.x.

Gallardo C., Soler A., Nieto R., Cano C., Pelayo V., Sánchez M.A., Pridotkas G., Fernandez-Pinero J., Briones V., Arias M. (2017) Experimental Infection of Domestic Pigs with African Swine Fever Virus Lithuania 2014 Genotype II Field Isolate. Transboundary and Emerging Diseases 64:300-304. DOI: 10.1111/tbed.12346.

Grear D.A., Samuel M.D., Scribner K.T., Weckworth B.V., Langenberg J.A. (2010) Influence of genetic relatedness and spatial proximity on chronic wasting disease infection among female white-tailed deer. Journal of Applied Ecology 47:532-540. DOI: 10.1111/j.1365-2664.2010.01813.x.

Goudet J. (1995). fstat (Version 1.2): a computer program to calculate F-statistics. Journal of Heredity 86, 485-486.

Hirsch B.T., Prange S., Hauver S.A., Gehrt S.D. (2013) Genetic relatedness does not predict racoon social network structure. Animal Behaviour 85:463-470. DOI: http://dx.doi.org/10.1016/j.anbehav.2012.12.011.

Hoffman J.I., Forcada J., Trathan P.N., Amos W. (2007) Female fur seals show active choice for males that are heterozygous and unrelated. Nature 445:912-914. DOI: 10.1038/nature05558.

Kaminski G., Brandt S., Baubet E., Baudoin C. (2005) Life-history patterns in female wild boars (Sus scrofa): mother-daughter postweaning associations. Canadian Journal of Zoology 83:474-480. DOI: 10.1139/z05-019.

Keuling O., Lauterbach K., Stier N., Roth M. (2010) Hunter feedback of individually marked wild boar Sus scrofa L.: dispersal and efficiency of hunting in northeastern Germany. European Journal of Wildlife Research 56:159-167. DOI: 10.1007/s10344-009-0296-x.

Lange M., Thulke H.-H. (2016) Elucidating transmission parameters of African swine fever through wild boar carcasses by combining spatio-temporal notification data and agent-based modelling. Stochastic Environmental Research and Risk Assessment:1-13. DOI: 10.1007/s00477-016-1358-8.

Manly B. F. J. (1997). Randomization, bootstrap and Monte Carlo methods in biology. $2^{\text {nd }}$ edn. London: Chapman and Hall.

Mejía-Salazar M.F., Goldizen A.W., Menz C.S., Dwyer R.G., Blomberg S.P., Waldner C.L., Cullingham C.I., Bollinger T.K. (2017) Mule deer spatial association patterns and potential implications for transmission of an epizootic disease. Plos One 12:e0175385.

Morelle K., Jezek M., Licoppe A., Podgorski T. (2019) Deathbed choice by ASF-infected wild boar can help find carcasses. Transboundary and Emerging Diseases 66:1821-1826. DOI: 10.1111/tbed.13267.

Morelle K., Bubnicki J., Churski M., Gryz J., Podgórski T., Kuijper D.P.J. (2020) Disease-Induced Mortality Outweighs Hunting in Causing Wild Boar Population Crash After African Swine Fever Outbreak. Frontiers in Veterinary Science 7. DOI: 10.3389/fvets.2020.00378. 
Nakagawa S., Johnson P.C.D., Schielzeth H. (2017) The coefficient of determination $R^{2}$ and intra-class correlation coefficient from generalized linear mixed-effects models revisited and expanded. Journal of The Royal Society Interface 14:20170213. DOI: doi:10.1098/rsif.2017.0213.

Nurmoja I., Schulz K., Staubach C., Sauter-Louis C., Depner K., Conraths F.J., Viltrop A. (2017) Development of African swine fever epidemic among wild boar in Estonia - two different areas in the epidemiological focus. Scientific Reports 7:12562. DOI: 10.1038/s41598-017-12952-w.

Peakall R., Smouse P. E. (2006). genalex 6: genetic analysis in Excel. Population genetic software for teaching and research. Molecular Ecology Notes 6, 288-295.

Pepin K.M., VerCauteren K.C. (2016) Disease-emergence dynamics and control in a socially-structured wildlife species. Scientific Reports 6:25150. DOI: 10.1038/srep25150

Pepin K.M., Golnar A., Podgórski T. (2021) Social structure defines spatial transmission of African swine fever in wild boar. Journal of The Royal Society Interface 18:20200761. DOI: doi:10.1098/rsif.2020.0761.

Pepin K.M., Golnar A.J., Abdo Z., Podgórski T. (2020) Ecological drivers of African swine fever virus persistence in wild boar populations: Insight for control. Ecology and Evolution 10:2846-2859. DOI: 10.1002/ece3.6100.

Pepin K.M., Davis A.J., Beasley J., Boughton R., Campbell T., Cooper S.M., Gaston W., Hartley S., Kilgo J.C., Wisely S.M., Wyckoff C., VerCauteren K.C. (2016) Contact heterogeneities in feral swine: implications for disease management and future research. Ecosphere 7:e01230. DOI: 10.1002/ecs2.1230.

Pietschmann J., Guinat C., Beer M., Pronin V., Tauscher K., Petrov A., Keil G., Blome S. (2015) Course and transmission characteristics of oral low-dose infection of domestic pigs and European wild boar with a Caucasian African swine fever virus isolate. Archives of Virology 160:1657-1667. DOI: 10.1007/s00705-015$2430-2$.

Podgorski T., Bas G., Jedrzejewska B., Sonnichsen L., Sniezko S., Jedrzejewski W., Okarma H. (2013) Spatiotemporal behavioral plasticity of wild boar (Sus scrofa) under contrasting conditions of human pressure: primeval forest and metropolitan area. Journal of Mammalogy 94:109-119. DOI: 10.1644/12-mamm-a-038.1.

Podgórski T., Śmietanka K. (2018) Do wild boar movements drive the spread of African Swine Fever? Transboundary and Emerging Diseases 65:1588-1596. DOI: doi:10.1111/tbed.12910.

Podgórski T., Scandura M., Jędrzejewska B. (2014a) Next of kin next door - philopatry and socio-genetic population structure in wild boar. Journal of Zoology 294:190-197. DOI: 10.1111/jzo.12167.

Podgórski T., Apollonio M., Keuling O. (2018) Contact rates in wild boar populations: Implications for disease transmission. The Journal of Wildlife Management 82:1210-1218. DOI: doi:10.1002/jwmg.21480.

Podgórski T., Borowik T., Łyjak M., Woźniakowski G. (2020) Spatial epidemiology of African swine fever: Host, landscape and anthropogenic drivers of disease occurrence in wild boar. Preventive Veterinary Medicine 177:104691. DOI: https://doi.org/10.1016/j.prevetmed.2019.104691.

Podgórski T., Lusseau D., Scandura M., Sönnichsen L., Jędrzejewska B. (2014b) Long-Lasting, Kin-Directed Female Interactions in a Spatially Structured Wild Boar Social Network. Plos One 9:e99875.

Poteaux C., Baubet E., Kaminski G., Brandt S., Dobson F.S., Baudoin C. (2009) Socio-genetic structure and mating system of a wild boar population. Journal of Zoology 278:116-125. DOI: 10.1111/j.14697998.2009.00553.x.

Prévot C., Licoppe A. (2013) Comparing red deer (Cervus elaphus L.) and wild boar (Sus scrofa L.) dispersal patterns in southern Belgium. European Journal of Wildlife Research 59:1-9. DOI: 10.1007/s10344-013-07329 . 
Probst C., Globig A., Knoll B., Conraths F.J., Depner K. (2017) Behaviour of free ranging wild boar towards their dead fellows: potential implications for the transmission of African swine fever. Royal Society Open Science 4:170054. DOI: 10.1098/rsos.170054.

Probst C., Gethmann J., Amendt J., Lutz L., Teifke J. P., Conraths F. J. (2020) Estimating the Postmortem Interval of Wild Boar Carcasses. Veterinary Sciences 7:6.

Queller D.C., Goodnight K.F. (1989). Estimating realtedness using genetic markers. Evolution 43:258-275. DOI: https://doi.org/10.1111/j.1558-5646.1989.tb04226.x.

R Core Team (2020). R: A language and environment for statistical computing. R Foundation for Statistical Computing, Vienna, Austria. https://www.R-project.org/.

Raymond M., Rousset F. (1995). Population genetics software for exact tests and ecumenicism. Journal of Heredity. 86, 248-249. https://doi.org/10.1093/oxfordjournals.jhered.a111573

Rice W. (1989). Analysing tables of statistical tests. Evolution 43, 223-225.

Riley S. (2007) Large-Scale Spatial-Transmission Models of Infectious Disease. Science 316:1298-1301. DOI: 10.1126 /science.1134695.

Sah P., Mann J., Bansal S. (2018) Disease implications of animal social network structure: A synthesis across social systems. Journal of Animal Ecology 87:546-558. DOI: https://doi.org/10.1111/1365-2656.12786.

Taylor R.A., Podgórski T., Simons R.R.L., Ip S., Gale P., Kelly L.A., Snary E.L. (2021) Predicting spread and effective control measures for African swine fever-Should we blame the boars? Transboundary and Emerging Diseases 68:397-416. DOI: https://doi.org/10.1111/tbed.13690.

Vander Wal E., Paquet P.C., AndrÉS J.A. (2012) Influence of landscape and social interactions on transmission of disease in a social cervid. Molecular Ecology 21:1271-1282. DOI: 10.1111/j.1365-294X.2011.05431.x.

VanderWaal K.L., Ezenwa V.O. (2016) Heterogeneity in pathogen transmission: mechanisms and methodology. Functional Ecology 30:1606-1622. DOI: https://doi.org/10.1111/1365-2435.12645.

Woźniakowski G., Kozak E., Kowalczyk A., Łyjak M., Pomorska-Mól M., Niemczuk K., Pejsak Z. (2016) Current status of African swine fever virus in a population of wild boar in eastern Poland (2014-2015). Archives of Virology 161:189-195. DOI: 10.1007/s00705-015-2650-5.

Yang A., Schlichting P., Wight B., Anderson W.M., Chinn S.M., Wilber M.Q., Miller R.S., Beasley J.C., Boughton R.K., VerCauteren K.C., Wittemyer G., Pepin K.M. (2020) Effects of Social Structure and Management on Risk of Disease Establishment in Wild Pigs. Journal of Animal Ecology n/a. DOI: https://doi.org/10.1111/1365-2656.13412.

Zuur A., Ieno, E.N., Walker, N., Saveliev, A.A., Smith, G.M. (2009) (Eds.) Mixed Effects Models and Extensions in Ecology with R. 574 pp. Springer-Verlag New York.

Table 2. Coefficients of the models explaining variation in ASF infection risk. Each model is formulated to test predictions of the hypothesis H3 outlined in Table 1 (model 1 and 2: P3.1; model 3: P3.2; model 4: P3.3.

\begin{tabular}{llllll}
\hline Model & $\mathrm{N}$ & Coefficient & Estimate $(95 \% \mathrm{CI})$ & $\mathrm{p}$-value & $R^{2}$ \\
\hline 1 & 55 & Intercept & $-3.57(-6.65 ;-1.72)$ & 0.004 & 0.76 \\
& & Proportion of infected $0-2 \mathrm{~km}$ & $6.80(4.03 ; 11.30)$ & $<0.001$ & \\
& \multirow{2}{*}{63} & Relatedness to infected $<10 \mathrm{~km}$ & $-0.94(-8.81 ; 9.00)$ & 0.823 & \\
& Intercept & $-0.76\left(-1.59 ;-0.3^{-4}\right)$ & 0.058 & 0.27 \\
& & Proportion of infected $2-5 \mathrm{~km}$ & $1.29(0.08 ; 2.55)$ & 0.039 & \\
& \multirow{2}{*}{96} & Relatedness to infected $<10 \mathrm{~km}$ & $4.16(0.33 ; 8.64)$ & 0.046 & \\
& & Intercept & $-1.13(-1.99 ;-0.33)$ & 0.007 & 0.16 \\
& & Proportion of infected $5-10 \mathrm{~km}$ & $2.01(0.75 ; 3.36)$ & 0.002 &
\end{tabular}




\begin{tabular}{llllll}
\hline Model & $\mathrm{N}$ & Coefficient & Estimate $(95 \% \mathrm{CI})$ & $\mathrm{p}$-value & $R^{2}$ \\
\hline \multirow{3}{*}{4} & \multirow{2}{*}{276} & Relatedness to infected $<10 \mathrm{~km}$ & $2.13(-1.11 ; 5.51)$ & 0.201 & \\
& Intercept & $-1.56(-2.05 ;-1.08)$ & $<0.001$ & 0.03 \\
& Proportion of infected $>10 \mathrm{~km}$ & $1.50(-0.25 ; 3.23)$ & 0.085 & \\
& Relatedness to infected $>10 \mathrm{~km}$ & $1.91(-0.92 ; 4.79)$ & 0.187 & \\
\hline
\end{tabular}

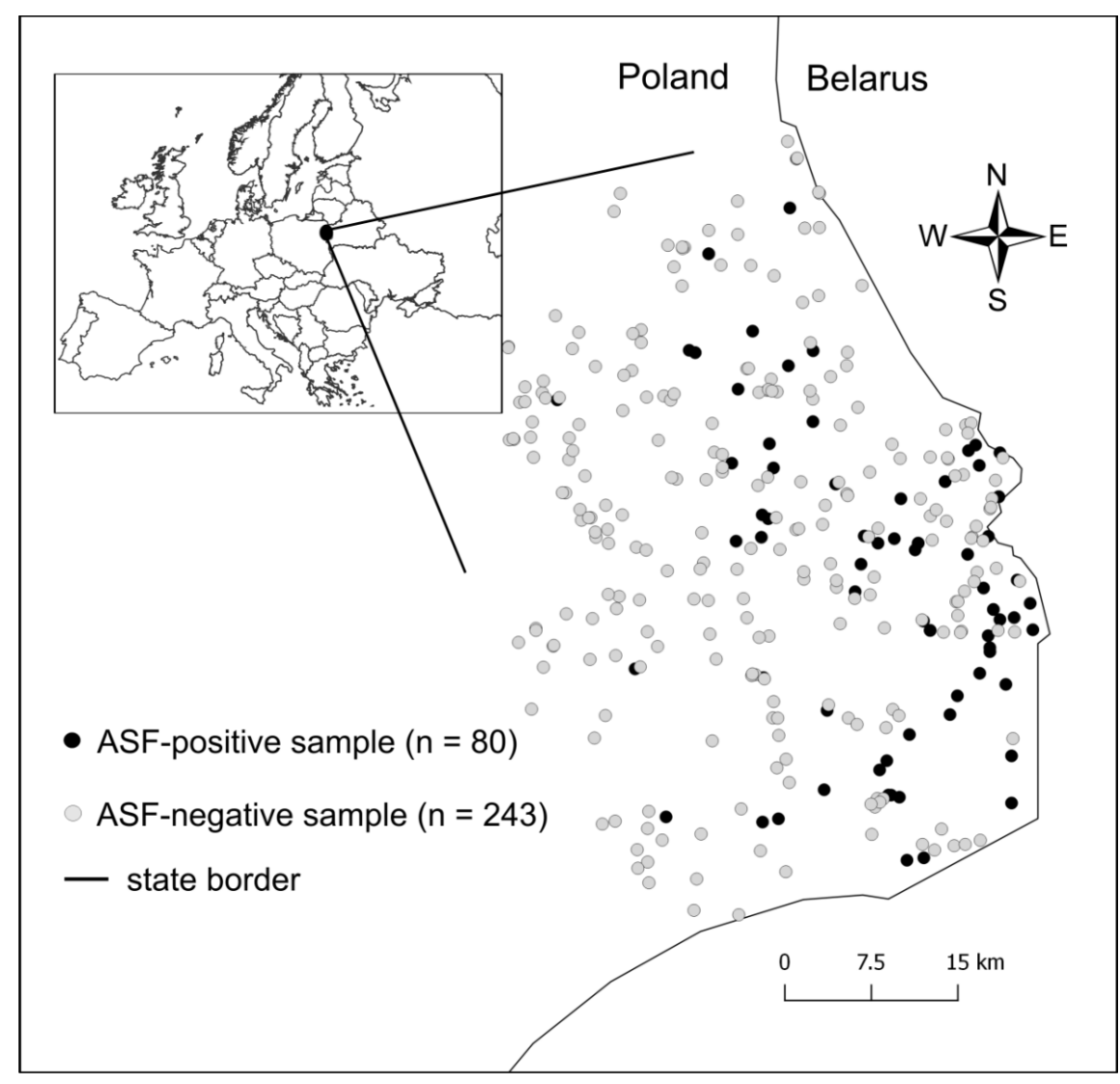

Fig. 1. Distribution of wild boar samples used in the study. 


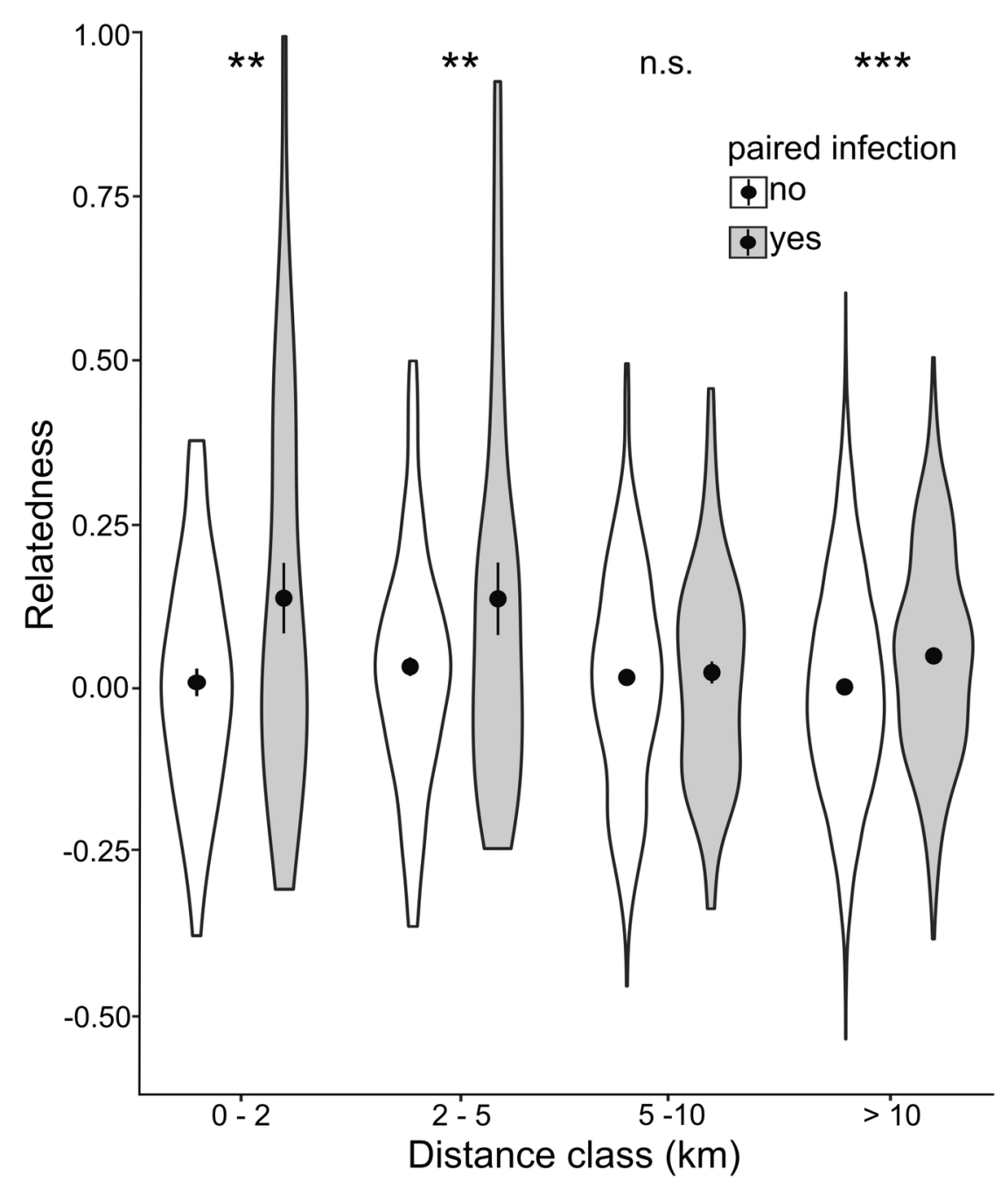

Fig. 2. Distribution of relatedness values among pairs of individuals with or without paired ASF infection (both individuals ASF positive vs. one/none individual ASF positive) across four distance classes (0-2km: 78 dyads; 2-5km: 183 dyads; 5-10km: 511 dyads; >10km: 6339 dyads). The violin plots show smoothed histogram of the data with mean (circle) and standard error (horizontal line). Statistical significance of the differences between the means $\left({ }^{* *} \mathrm{p}<0.001,{ }^{* *} \mathrm{p}<0.01\right.$, n.s. $\left.\mathrm{p}>0.05\right)$ was obtained with randomization tests. 

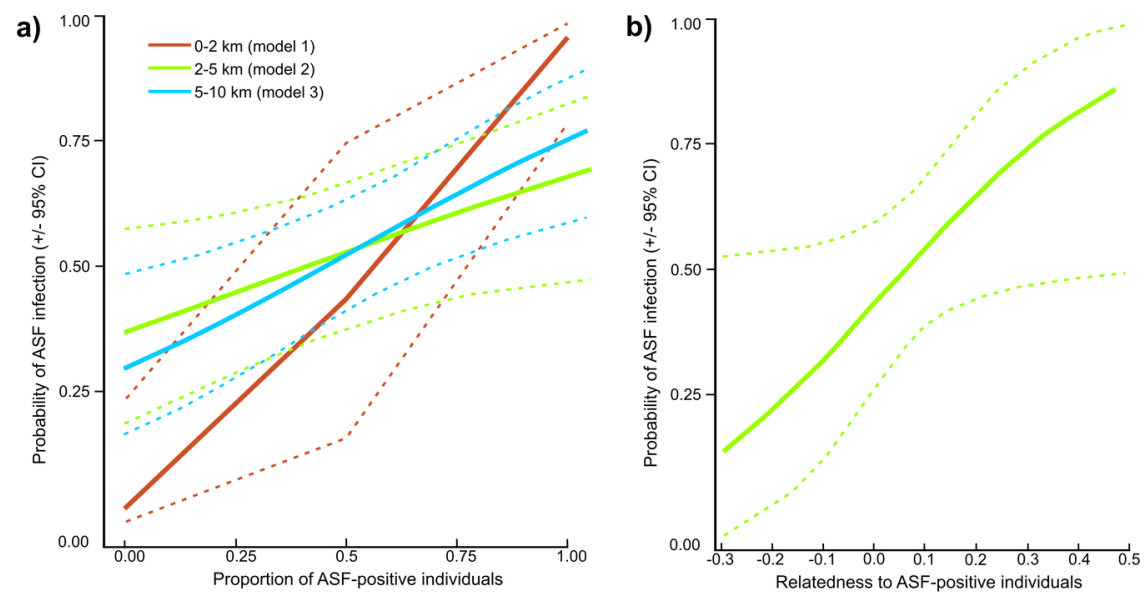

Fig. 3. Relationship between a) proportion of ASF-infected individuals and b) relatedness to ASF-infected individuals and ASF infection risk predicted across three proximity categories varying in frequency of contact (0-2km: high-contact; 2-5km: medium contact; 5-10km: low-contact). Solid lines represent the effects estimated with generalized linear model (Table 2) and dashed lines represent 95\% confidence limits around the model estimates. Only significant effects are plotted. 